\title{
RECURRENT PAROTITIS IN CHILDREN: WHEN SHOULD WE SUSPECT OF SJOGREN SYNDROME?
}

Lorena Torres Giacomin ${ }^{1}$, Paulo Rogério Julio ${ }^{1}$, Roberto Marini ${ }^{1}$, Otavio Alves Garcia Junior ${ }^{1}$, Simone Appenzeller ${ }^{1, \star}$, Rebecca Maunsell ${ }^{1}$

1.Universidade Estadual de Campinas, Campinas (SP), Brazil.

*Corresponding author: appenzellersimone@gmail.com

\section{BACKGROUND}

To compare the epidemiological, clinical, and imaging profile of children with primary Sjögren's syndrome (pSS) and patients with juvenile recurrent parotitis (JRP).

\section{MATERIALS AND METHODS:}

We retrospectively collected data from patients presenting with recurrent parotitis in our service. Variables including age at recurrent parotitis onset, number of parotitis episodes per year, average number of the years of recurrent parotitis before the definitive diagnosis, definitive diagnosis and presence of systemic features were compared between groups.

\section{RESULTS}

We identified 15 patients [9 (60\%) patients with pSS and 8 (40\%) with JRP]. The mean age at first episode of parotitis was significantly higher in pSS (7vs 3.4 years; $p=0.02$ ). We did not observe differences in the age at diagnosis, mean number of episodes per year, clinical characteristics of the acute episodes of parotitis and presence of systemic features between groups.

\section{CONCLUSION}

Recurrent parotitis is the most common clinical presentation in childhood-onset pSS. Although rare, pSS should be considered as a differential diagnosis in recurrent parotitis.

\section{FUNDING}

Conselho Nacional de Desenvolvimento Científico (CNPq) 304255/2015-7; Coordenação de Aperfeiçoamento de Pessoal de Nível Superior - Brasil (CAPES) 001. 